\title{
Biological Therapies for Atopic Dermatitis: A Systematic Review
}

\author{
Shuying Zhou ${ }^{\mathrm{a}}$ Fei $\mathrm{Qi}^{\mathrm{b}}$ Yue Gong $^{\mathrm{a}}$ Jinping Zhang ${ }^{\mathrm{a}}$ Binghua Zhu ${ }^{\mathrm{a}}$ \\ ${ }^{a}$ Department of Dermatology, The 305 Hospital of PLA, Beijing, China; ${ }^{b}$ Capital Medical University \\ Affiliated with Beijing Chaoyang Hospital, Beijing, China
}

\section{Keywords}

Atopic dermatitis · Biologicals · Immunotherapy

\begin{abstract}
Background: Atopic dermatitis (AD) is a widely acquired, relapsing inflammatory skin disease. Biologics are now widely used in patients with moderate-to-severe AD. Objective: This work aims to summarize both label and off-label biologics on AD treatment in phase II and phase III stages, and compile evidence on the efficacy of the most-studied biologics. Methods: We conducted a comprehensive literature search through PubMed, EMBASE, and ClinicalTrials.gov to identify all documented biological therapies for AD. The criteria were further refined to focus on those treatments with the highest evidence level for $A D$ with at least one randomized clinical trial supporting their use. Only studies or articles published in English were enrolled in this study. Findings: Primary searches identified 525 relevant articles and 27 trials. Duplicated articles and papers without a full text were excluded. Only completed trials were enrolled. We included 28 randomized controlled trials, 4 unpublished trials, 2 observational studies, and 1 meta-analysis. Eight kinds of biologics, including IL-4/IL-13 inhibitors, JAK inhibitors, anti-IL-13 anti-
\end{abstract}

S.Z. and F.Q. contributed equally to this work.

karger@karger.com
www.karger.com/drm

bodies, anti-IL-22 antibodies, anti-IL-33 antibodies, thymic stromal lymphopoietin inhibitor (TSLP), OX40 antibodies, and H4R-antagonists were included in this work. Dupliumab, as the most widely used and investigated biologic, was reported in 1 meta-analysis and 4 trials exploring its long-term use and application in both adults and pediatric patients. Besides dupilumab, four other IL-4/IL-13 inhibitors recruited were all randomized, clinical trials at phase $2-3$ stage. Six different kinds of JAK inhibitors were summarized with strong evidence revealing their significant therapeutic effects on AD. There were 3 trials for nemolizumab, an anti-IL-13 antibody, all of which were in the phase 2 clinical trial stage. Results showed nemolizumab could be another alternative therapy for moderate-to-severe AD with long-term efficiency and safety. Conclusion: The biological therapies with the most robust evidence on efficacy and long-term safety for AD treatment include dupilumab, barcitinib, abrocitinib, and delgocitinib. Most of the biologics mentioned in this review were still at the exploratory stage. This review will help practitioners advise patients seeking suitable biological therapies and offer experimental study directions for treatment.

(C) 2021 S. Karger AG, Basel

The Third Clinical Teaching Hospital

Capital Medical University Affiliated with Beijing Chaoyang Hospital

8 Gongren Tiyuchang Nanlu, Chaoyang District, Beijing 100020 (China) qifeiphaedra@gmail.com

Binghua Zhu

Department of Dermatology

The 305 Hospital of PLA

17 Wenjinjie, Xicheng District, Beijing 100017 (China)

zbh305army@126.com 


\section{Introduction}

Atopic dermatitis (AD) is a chronically relapsing inflammatory skin disease that affects $2.1-4.9 \%$ of adults and $20 \%$ of children $[1,2]$. Nearly one-third of children and half of adult patients with $\mathrm{AD}$ have moderate or severe diseases, which may have poor responses to topical treatment and phototherapy [3]. A recently published cohort study summarized the burden of skin and subcutaneous diseases in the USA in the last 30 years described $\mathrm{AD}$ as the second most prevalent skin disease in the country in 2017, excluding keratinocyte carcinoma and malignant melanoma [4].

Conventional management of AD consists of phototherapy and treatments that comprise cyclosporine A, topical application of calcineurin inhibitors (including tacrolimus and pimecrolimus), and corticosteroids (in both topical and systematic use) [5]. Targeted therapies for $\mathrm{AD}$, including IL-4/13 inhibitors, JAK inhibitors, and IL-13 inhibitors used in both topical and systematic ways, have been introduced to moderate-to-severe AD patients with systemic treatment resistance as a novel therapeutic method in recent years [6]. Numerous biologics are now under investigation or at an exploratory stage. In contrast, some of the biologics are currently in phase 2 and 3 clinical trials, and a minority of therapies, such as crisaborole ointment and dupilumab, approved by the US Food and Drug Administration (FDA), have been further devoted to clinical application in the real-world setting [7].

Given the pleomorphic presentations, different prognosis, the burden of comorbidities, as well as contraindications to both the conventional systemic treatment and targeted therapy, an individualized treatment and management plan should be taken into account [8]. Nanotechnology-based therapeutics have been investigated as a possible treatment for $\mathrm{AD}$, which can prepare for individualized treatment of $\mathrm{AD}$ in the aspects of drug delivery, controlled release, dosage, and skin penetration [9]. Compared to currently available drugs, nanotechnologies enable better bioavailability, transdermal delivery, and better dose control as the carriers of biologics. They can also act as systemic drugs through a topical application with a lower incidence of adverse events [10] and provide higher treatment efficacy.

As for many clinicians, understanding the efficacy, safety, and dosage strategy of different biologics is challenging. Although many published systematic reviews and meta-analyses of randomized clinical trials have included some novel therapies and quantitative analysis of the relative effectiveness and safety of biologics, a review

Biological Therapies for Atopic

Dermatitis covering most biologics currently under investigation is still needed. Herein, we reviewed the literature and trials on the use of biologics to treat $\mathrm{AD}$ in pediatric and adult patients. Moreover, we further predicted the possible future study direction of trials in therapeutic AD biologics, which may offer some instructions to clinicians who would like to try the biologics mentioned in this review.

\section{Methods}

This study followed the "Preferred Reporting Items for Systematic Reviews and Meta-analyses" (PRISMA) guidelines [11]. We firstly identified all biological methods in the treatment of $\mathrm{AD}$ through UpToDate and Google Scholar. A comprehensive search was conducted in MEDLINE (using PubMed), EMBASE, Chinese Trial Database (CHICTR), and Clinical Trials.gov by two investigators to recruit all relevant articles and trials about $\mathrm{AD}$ biologics. The search terms were defined with the help of MESH and Emtree in PubMed and EMBASE, respectively. The search strategy was constructed to find publications containing at least one term from each of the two following search blocks: ("biological therapy" or "immunomodulatory treatment") and ("atopic dermatitis" or "eczema"). For further research, we used some exact names of some specific biologics: "dupilumab," "lebrikizumab," "nemolizumab." Two individuals evaluated the titles and abstracts. Only English language original studies performed on human subjects were enrolled in this review (Fig. 1).

\section{Results}

Five hundred twenty-five relevant articles were found in PubMed and EMBASE, and 58 trials were recovered from ClinicalTrials.gov and CHICTR. Duplicated articles and papers without a full text were excluded. Thirty-three published articles, and 4 unpublished trials presented 8 kinds of biologics that were found after full-text evaluation. Biologics with at least one trial supporting their use in $\mathrm{AD}$ were included for qualitative analysis. This review comprised 28 randomized controlled trials and 4 unpublished trials (online suppl. Table 1; for all online suppl. material, see www.karger.com/doi/10.1159/000514535).

We found a total of 8 kinds of biologics, including IL-4/IL-13 inhibitors, JAK inhibitors, anti-IL-13 antibodies, anti-IL-22 antibodies, anti-IL-33 antibodies, thymic stromal lymphopoietin inhibitor (TSLP), OX40 antibodies, and H4R antagonist. Among these biologics, dupilumab is now the most widely studied and used IL-4/IL-13 inhibitor and has been approved in the real world. Other biologics are currently in phase 2 and phase 3 clinical trials. Further studies are necessary to evaluate their efficacy and safety in $\mathrm{AD}$ treatment. 
Fig. 1. Study flowchart illustrating the articles and trials.

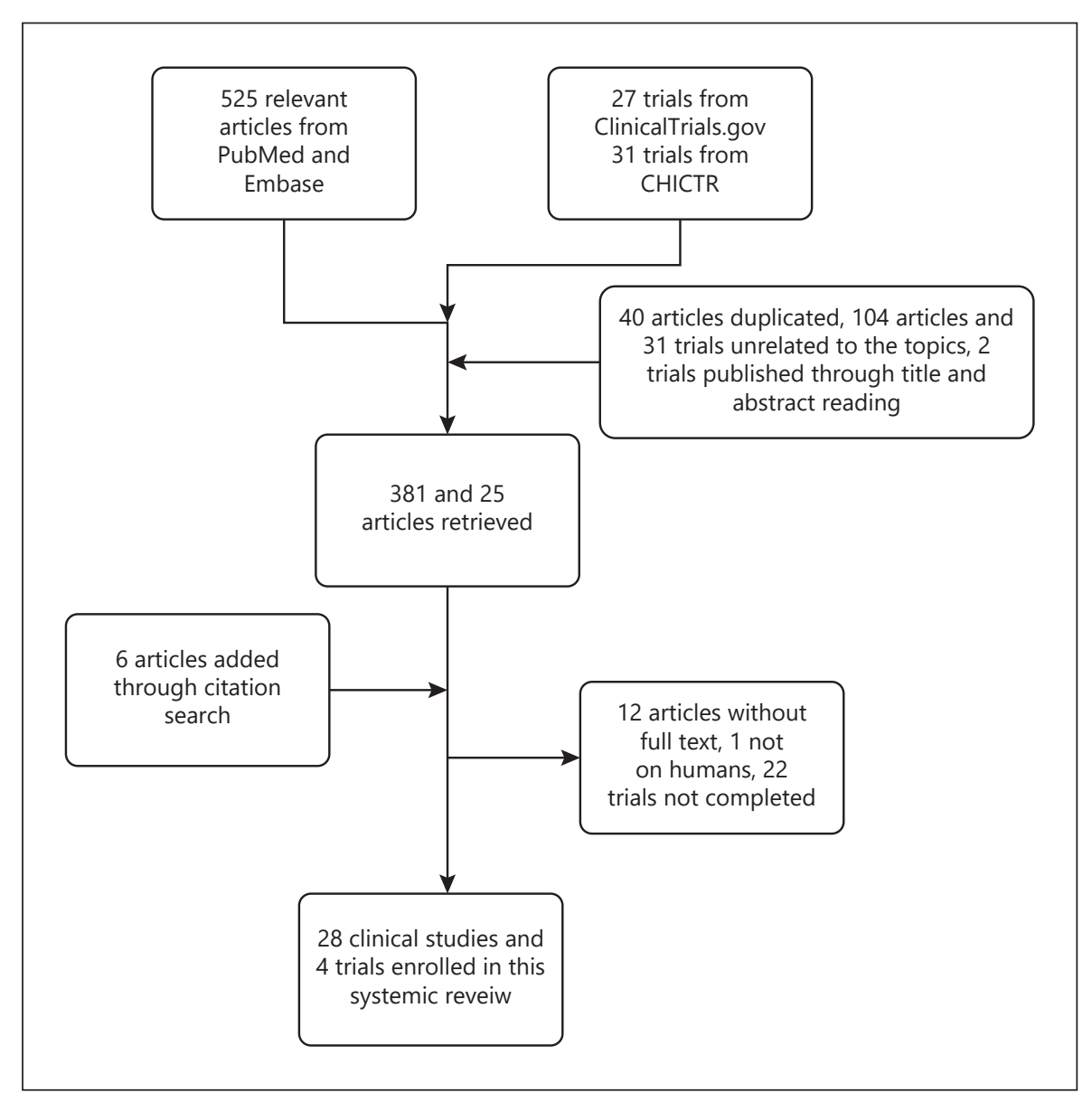

\section{IL-4/IL-13 Inhibitor}

$\mathrm{AD}$ is now believed to be characterized by the overexpression of Thelper 2 (Th2) cytokines, including IL-4 and IL-13 [12]. IL-4 is thought to work as a critical amplifier of type 2 immunity by recruiting CD4+ Th2 cells, while IL-13 is regarded as a primary disease-inducing effector cytokine [13]. IL-4 and IL-13 can activate Th2 cells, inducing myeloid and atopic dendritic cell differentiation, activating B cells, stimulating IgE class switching, and promoting eosinophil recruitment [14]. Furthermore, IL-4 and IL-13 have been recognized as having a strong connection with $\mathrm{AD}$ disease activity [13]. The blockade of IL4/13 is effective in reducing the Th2 response [15].

\section{Dupilumab}

Dupilumab is a fully human monoclonal IgG4 antibody that inhibits IL- 4 and IL-13 signal transduction by binding the shared $\alpha$-subunit of the IL-4 receptor [16]. A previous study also presented a significant deduction on serum levels of CCL17 (or thymus and activation-regu- lated chemokine), a key regulator of Th2-mediated immunity, and a specific and objective biomarker reflecting $\mathrm{AD}$ disease activity $[15,17]$. It is now approved by the US FDA and used in the real world for moderate-to-severe $\mathrm{AD}$ patients.

Hamilton et al. [14] reported that dupilumab improved $\mathrm{AD}$ in a dose-dependent manner by comparing $\mathrm{AD}$ patients treated weekly with 150 or $300 \mathrm{mg}$ of dupilumab or placebo. Genes that are upregulated in AD lesions decreased when treated with dupilumab, and the decrements were correlated with clinical scores. When patients received $300 \mathrm{mg}$ of dupilumab for 4 weeks, a significant reduction in the mRNA expression of genes related to hyperplasia (K16 and MKI67), T cells, and dendritic cells (CD1b and CD1c), as well as potent inhibition of Th2-associated chemokines (CCL17, CCL18, CCL22, and CCL26), was observed compared with the baseline.

Halling et al. [18] recently reviewed the real-world data of the efficacy and safety of dupilumab on 3,303 adolescents and adults with moderate-to-severe $\mathrm{AD}$ in 22 
studies. After giving a 600-mg loading dose of dupilumab and a following 300-mg maintenance dose every second week, dupilumab presented as a successful and well-tolerated therapy for AD. No short-term safety signals except conjunctivitis, blepharitis, injection site reactions, and herpes simplex virus infection were found in these studies.

In a study by Ferrucci et al. [19], the efficacy and safety of dupilumab were further evaluated. This trial also showed that an onset before the age of 18 years or hypereosinophilia absence in $\mathrm{AD}$ patients were identified as significant predictive parameters for a positive response to dupilumab in terms of the Eczema Area and Severity Index (EASI)-75 in week 4 but not by week 16 . This suggested that an early $\mathrm{AD}$ onset and absence of hypereosinophilia may be the markers of early response to dupilumab.

As for its long-term safety and efficacy, Deleuran et al. [20] conducted a follow-up study on patients who received $300 \mathrm{mg}$ dupilumab weekly for up to 76 weeks, among which $92.9 \%$ of patients showed positive efficient outcomes. Common adverse events included nasopharyngitis, conjunctivitis, and injection-site reactions. This study supported the role of dupilumab as a continuous long-term treatment for patients with moderate-to-severe $A D$. Further study should focus on the efficacy of receiving dupilumab $\geq 76$ weeks and $300 \mathrm{mg}$ every 2 weeks (Q2W).

A 16-week randomized, double-blind study focused on adolescent $\mathrm{AD}$ patients aged between 12 and 18 years was carried out by Cork et al. [21]. Patients received a dupilumab dose ( 2 or $4 \mathrm{mg} / \mathrm{kg}$ ) weekly for 4 weeks with an 8-week safety follow-up and a 52-week, open-label extension study. All enrolled subjects had positive responses to dupilumab. EASI showed improvements until week 52, and the most common adverse effect was nasopharyngitis. From this trial, dupilumab showed long-term safety and efficacy in adolescent patients aged from 12 to 18 years.

Another trial investigating the long-term safety and efficacy of dupilumab in children aged $\geq 6$ to $<12$ years with severe AD was reported recently [22]. Single doses of dupilumab 2 or $4 \mathrm{mg} / \mathrm{kg}$ were given to enrolled pediatric $\mathrm{AD}$ patients weekly for 4 weeks. Results showed a nonlinear, target-mediated pharmacokinetic concentration of dupilumab. Single-dose dupilumab rapidly improved $\mathrm{AD}$ with further improvement through to week 52 . The mean EASI had improved in weeks 2 and 52.

In summary, dupilumab is a relatively safe and efficient biologic therapy in both adults and children $\mathrm{AD}$ pa-

Biological Therapies for Atopic

Dermatitis tients. It is a relatively well-investigated biologic with few adverse effects. It has been used in the real world, whilst long-term safety and efficacy still need to be explored.

\section{Lebrikizumab}

Lebrikizumab is a high-affinity, monoclonal IL-13 antibody. It prevents the formation of the IL-13Ra1/IL$14 \mathrm{Ra}$ heterodimer receptor signaling complex [23] and has been previously used to treat asthma [24]. Based on the involvement of IL-13 in multiple pathways that are important to $\mathrm{AD}$ pathogenesis, lebrikizumab may represent a novel targeted therapy in AD [25].

In a phase 2 trial conducted by Simpson et al. [25], 5 groups of people were respectively given lebrikizumab as a 125 -mg single dose (SD), 250-mg SD, $125 \mathrm{mg}$ once every 4 weeks (Q4W), and placebo Q4W for 12 weeks. In week 12 , more patients who received lebrikizumab $125 \mathrm{mg}$ Q4W achieved EASI-50 compared with the placebo and lebrikizumab SD groups.

In the trial by Guttman-Yassky et al. [26], 4 groups of AD patients received, respectively, placebo Q2W, $125 \mathrm{mg}$ Q4W with a 250-mg loading dose, $250 \mathrm{mg}$ Q4W with a 500-mg loading dose, and $250 \mathrm{mg}$ Q2W with a $500-\mathrm{mg}$ loading dose at baseline and week 2 . After 16 weeks of treatment, lebrikizumab showed dose-dependent efficacy in adult moderate-to-severe $\mathrm{AD}$ patients compared to placebo. A few adverse effects presented, including injectsite reactions, herpesvirus infections, and conjunctivitis.

In summary, the clinical application of lebrikizumab on $\mathrm{AD}$ treatment is still subject to phase 2 clinical trials. For long-term safety and efficacy, more studies should be conducted in the future $[27,28]$.

\section{Tralokinumab}

Tralokinumab is a fully human $\operatorname{IgG}_{4} \mathrm{mAb}$ that specifically binds to IL-13 [29]. In a phase $2 \mathrm{~b}$ study conducted by Wollenberg et al. [30], 204 adults were assigned $1: 1: 1: 1$ to receive 45,150 , or $300 \mathrm{mg}$ of subcutaneous tralokinumab, or placebo, Q2W for 12 weeks with topical glucocorticoids. Improvement of EASI and investigator's global assessment (IGA) were evaluated in week 12. Results showed that $300 \mathrm{mg}$ of tralokinumab led to a significant improvement in EASI and a greater percentage of participants achieved an IGA response. Biomarkers of IL-13 activity were also tested and demonstrated more significant responses in IL-13 activity. Adverse events were commonly included in upper respiratory tract infections.

A phase 3 trial, concluded in 2020 and sponsored by LEO Pharma [31], enrolled 794 moderate-to-severe AD 
patients. Subjects were randomized 3:1 to initial treatment with tralokinumab $300 \mathrm{mg}$ Q2W or placebo for 16 weeks. In week 16, subjects were re-randomized 2:2:1 to maintenance with $300 \mathrm{mg}$ Q2W, $300 \mathrm{mg}$ Q4W, or placebo until week 52. IGA and the percentage achieving EASI-75 were used to access the efficacy. In week 16, $33.2 \%$ of patients who received tralokinumab achieved EASI-75 compared with $11.4 \%$ in the placebo group. In the maintenance period through to 52 weeks, patients who received the biologic Q2W showed greater improvement in IGA, and more patients achieved EASI-75 compared to the Q4W group and placebo.

In summary, tralokinumab is currently in a phase 3 clinical trial, in which it has proved to be an acceptable and efficient therapeutic method for AD patients. Longterm observation for 52 weeks has demonstrated safety, with fewer adverse events in clinical use. More trials in patients younger than 18 years old should be conducted.

\section{JAK Inhibitors}

The JAK (Janus kinase)-signal transducer and activator of transcription (STAT) signaling, and spleen tyrosine kinase pathways have been implicated in AD and other autoimmune and inflammatory diseases [32, 33]. Both pathways are involved in modulating multiple immune pathways involved in AD, including Th2 (IL-4, IL-5, IL-6, IL-10, IL-13, IL-31) [34]. Ciechanowicz et al. [35] summarized the cutaneous manifestations of JAK-STAT mutations and analyzed the effect of JAK inhibitors in psoriasis, $\mathrm{AD}$, and vitiligo. Most JAK inhibitors are used orally and topically. However, the application of JAK inhibitors on AD treatment is still in phase 2 and 3 trials. More studies exploring the adverse effects and efficacy should be conducted in the future.

\section{Tofacitinib}

Tofacitinib is a small-molecule JAK inhibitor that can directly inhibit cytokines such as IL-4 [36] and decrease the JAK-STAT signal in keratinocytes [37]. Previous phase $2 \mathrm{~b}$ and phase 3 studies have demonstrated the efficacy of tofacitinib in moderate-to-severe chronic plaque psoriasis [38-41].

In a study conducted by Bissonnette et al. [42], 35 mild-to-moderate $\mathrm{AD}$ patients were given $2 \%$ tofacitinib twice daily compared with 34 controls. After 4 weeks of treatment, $81.7 \%$ of patients using tofacitinib showed improvement in the EASI versus improvement in $29.9 \%$ of controls who received vehicle ointment. These results showed the efficacy of tofacitinib in $\mathrm{AD}$ treatment. Tofacitinib is still in the phase 2 a clinical tri- al stage, whereas more clinical trials should be completed to evaluate the ointment's optimal therapeutic concentration and safety.

\section{Baricitinib}

Baricitinib is a first-generation oral selective JAK $1 / 2$ inhibitor [43]. It was approved for the treatment of rheumatoid arthritis by the FDA in 2018.

In 2018, Guttman-Yassky et al. [44] treated 124 moderate-to-severe $\mathrm{AD}$ patients with placebo, barcitinib 2 $\mathrm{mg}$, or baricitinib $4 \mathrm{mg}$ once daily for 16 weeks after applied topical corticosteroids (TCS) for 4 weeks. The primary outcome showed that more patients who received 4 mg of barcitinib achieved EASI-50 compared to placebo, which suggested a possibly novel efficient treatment for moderated-to-sever AD.

In 2 completed phase 3 clinical trials (NCT03334396 and NCT03334422) [45, 46], 660 and 615 adult moderate-to-severe $\mathrm{AD}$ patients were divided into 4 groups and administered placebo, 1,2 , and $4 \mathrm{mg}$ barcitinib once daily, respectively. After 16 weeks, patients showed a dosedependent improvement in lesions and pruritic feeling compared with the control group. In another recently completed study [47], investigators combined both $4 \mathrm{mg}$ barcitinib orally and TCS to treat moderate-to-severe AD. The results showed a significant improvement in EASI after 16 weeks compared with placebo.

Barcitinib is still at the phase 3 clinical trial stage. Although the above trials may indicate the efficacy in $\mathrm{AD}$ treatment, more studies should be conducted to evaluate the safety and long-term effectiveness [48]. Current ongoing trials are mainly focusing on assessing the possible application of barcitinib in children and adolescents [49].

\section{Abrocitinib}

Abrocitinib is an oral, once-daily JAK1 selective inhibitor, which modulates IL-4 and IL-13 and other cytokines involved in the pathogenesis of AD. It spares JAK2 inhibition, which minimizes the risk for neutropenia and anemia [50]. Oetjen et al. [51] found that the inhibition of neuronal JAK1 pathways can ameliorate pruritus.

A phase $2 \mathrm{~b}$ randomized clinical trial [52] assigned 267 adult moderate-to-severe $\mathrm{AD}$ patients $1: 1: 1: 1: 1$, respectively, to receive abrocitinib 200,100,30,10 mg, or placebo orally once a day for 12 weeks. The proportions of patients who achieved an IGA score of 0 (clear) and 1 (almost clear), and an improvement of 2 grades or more was evaluated. In week 12, only the 100- and 200-mg-dose groups achieved the previously set goals and found significant reductions of EASI. Abrocitinib was shown to be 
an effective short-term therapeutic method for moderateto-severe $\mathrm{AD}$ dermatitis.

In a multicenter, double-blind, randomized phase 3 trial (JADE MONO-1), AD patients aged above 12 years with an IGA score $\geq 3$ were enrolled and administrated 200 or $100 \mathrm{mg}$ of abrocitinib or placebo, respectively, once daily for 12 weeks. Results showed $40 \%$ of patients in the 100-mg group, and $63 \%$ of patients in the $200-\mathrm{mg}$ group achieved an EASI-75, which was greater than the placebo group [53].

Similar results were also shown in a JADE MONO-2 clinical trial conducted by Silverberg et al. [54], in which $37.3 \%$ of $\mathrm{AD}$ patients in the $100-\mathrm{mg}$ group and $34.9 \%$ in the 200-mg group achieved an EASI-90 improvement, with adverse effects such as decreases in platelet count (1.3\%) and thrombocytopenia (3.2\%) in the 200-mg group. The most commonly reported treatment-emergent adverse events included diarrhea, nausea, viral upper respiratory tract infection, headache, and dermatitis. The clinical application of abrocitinib is still in the phase 3 experimental stage. More studies should be conducted on its safety and long-term efficacy in the future.

\section{Upadacitinib}

Upadacitinib is an oral JAK1 inhibitor and is currently being investigated in the treatment of several immunemediated inflammatory diseases. It was approved in the treatment of rheumatoid arthritis in 2019. It is currently at the phase 3 clinical trial stage. In a 16-week trial investigated by Guttman-Yassky et al. [55], 4 groups of adult $\mathrm{AD}$ patients were respectively given upadacitinib oral monotherapy in 7.5-, 15-, or 30-mg doses, or placebo. In week 16, the percentage EASI improvement was evaluated to analyze the efficacy. The results indicated a doseresponse relationship for upadacitinib efficacy, and the once-daily 30-mg dose showed the greatest efficacy, with a reduction in pruritus and improvements in sleep. There has only been one clinical trial for upadacitinib. More studies should be conducted to further evaluate its effective dosage, safety, and long-term efficacy in pediatric and adult moderate-to-severe AD patients.

\section{Ruxolitinib}

Ruxolitinib (RUX) is a topical selective JAK1 and JAK2 inhibitor. In a phase 2 study [56], 307 adult AD patients with IGA 2 or 3 were equally randomized to apply RUX $1.5 \%$ twice daily (b.i.d.), $1.5 \%$ once daily (q.d.), $0.5 \%$ q.d., $0.15 \%$ q.d., vehicle, or $0.1 \%$ triamcinolone cream b.i.d. for 4 weeks. Results showed that in week 4 , the $1.5 \%$ b.i.d. group showed the greatest improvement in EASI (71.6\%) and IGA (38.0\%) versus vehicle. A rapid reduction in the itch numerical rating scale score appeared within $36 \mathrm{~h}$ and was sustained for 12 weeks. It is still under phase 2 clinical exploration. Two more ongoing trials $[57,58]$ are focusing on the efficacy and safety of RUX cream in adolescents and adults with $\mathrm{AD}$, while one is exploring the application in pediatric AD patients [59]. Further studies should focus on the long-term efficacy and safety and comparatively explore its efficacy with other topical $\mathrm{AD}$ treatment ointments.

\section{Delgocitinib}

Delgocitinib (JTE-052) is a novel, small-molecule, topical, non-selective JAK inhibitor [60] that is widely used in skin inflammation suppression [61], skin barrier dysfunction [62], and the reduction of IL-31-induced pruritus [63]. In a double-blind trial [64], moderate-to-severe $\mathrm{AD}$ patients aged 16 years or above were assigned $2: 1$ to receive $0.5 \%$ delgocitinib ointment or vehicle ointment, respectively, for 4 weeks, with a 24 -week extension period for $0.5 \%$ delgocitinib ointment treatment. In week 4 , the improvement of EASI was more remarkable in the delgocitinib group and sustained through the following 24week extensional treatment period with mild adverse events.

Nakagawa et al. [65] also demonstrated the long-term safety and efficacy of $0.5 \%$ delgocitinib ointment b.i.d. in a 52-week study. A total of $506 \mathrm{AD}$ patients were included with significant EASI improvement, and common adverse events included nasopharyngitis, contact dermatitis, acne, and application site folliculitis.

As for pediatric AD patients aged 2 through to 15 years, a phase 2 clinical study in Japanese patients was conducted by Nakagawa et al. [66] in which 0.25 and $0.5 \%$ delgocitinib ointment or vehicle ointment were equally administered to 3 groups of young patients for 4 weeks. The modified EASI scores in both delgocitinib groups were significantly reduced with mild adverse events. Delgocitinib has currently finished its phase 3 clinical trials in both children and adult AD patients. However, those trials were mostly conducted in Japanese patients. More investigations on patients from different races should be further explored.

\section{Anti-IL-31 Antibodies and Nemolizumab}

IL-31 plays a role in the pathogenesis of $\mathrm{AD}$ and the occurrence of pruritis. Compared with normal skin, higher IL-31 receptor A (RA) levels were expressed on epidermal keratinocytes in $\mathrm{AD}$ samples, which might contribute to the development of AD-induced skin in- 
flammation and pruritus [67]. Szegedi et al. [68] demonstrated that many IL-31-producing $\mathrm{T}$ cells coproduced IL-13 and, to a lesser extent, IL-22. They also showed that $\mathrm{T}$ cells in chronic AD skin produced cytokines like IL-31, which suggested a unique role of IL-31 in the pathogenesis of AD.

Nemolizumab (CIM331) is a humanized monoclonal antibody against IL-31 RA, which can bind IL-31 RA on several cells, including neurons, and may alleviate pruritus [69]. In a phase 2, randomized, double-blind, placebocontrolled study conducted by Ruzicka et al. [70], moderate-to-severe $\mathrm{AD}$ patients were assigned into 5 groups and given a subcutaneous nemolizumab dose of $0.1,0.5$, or $2.0 \mathrm{mg} / \mathrm{kg}$ of body weight or placebo every 4 weeks, or $2.0 \mathrm{mg} / \mathrm{kg}$ every 8 weeks. In week 12, the improvement in pruritus was evaluated through the visual analog scale, and EASI was used to describe the improvement of the skin lesions. Two hundred and sixteen patients completed the 12-week trials. All patients in the monthly dose group showed a significant improvement both in pruritis and EASI in a dose-dependent manner compared with the placebo group.

Mihara et al. [71] reported a similar phase 2 study and further conducted a 52-week extension period of nemolizumab treatment. They showed an improvement in both work productivity and pruritus-associated sleep disturbance for nemolizumab-treated patients, and the efficacy was sustained through to week 64, which suggested a relatively long-term efficacy and safety of this novel biologic.

Silverberg et al. [72] performed a 24-week study in which AD patients were given nemolizumab subcutaneous injections of 10, 30, and $90 \mathrm{mg}$ every 4 weeks versus placebo along with TCS application. The results showed improved EASI and IGA, and numeric rating scale itch scores peaked at the $30-\mathrm{mg}$ dose, with nasopharyngitis and upper respiratory tract infection as the most common adverse events.

The phase 2 clinical trials of nemolizumab have been completed. More ongoing studies are being performed to further evaluate the safety and long-term efficacy. Phase 3 studies in both adults and pediatric patients should be carried out in the future.

\section{Anti-IL-22 Antibodies and Fezakinumab}

Increased expression of Th22 cytokine IL-22 is a characteristic finding in AD. IL-22 plays an important pathogenic role in the initiation and development of $\mathrm{AD}$ [73]. Fezakinumab (ILV-094) is an IL-22 antagonist and was in an investigator-initiated phase $2 \mathrm{a}$ trial for $\mathrm{AD}$ treat- ment [74]. The randomized study enrolled 60 moderateto-severe $\mathrm{AD}$ patients and assigned them to either fezakinumab intravenously or placebo (2:1) with a loading dose of $600 \mathrm{mg}$ on day 0 , followed by $300 \mathrm{mg}$ Q2W. The last dose was given in week 10 . In week 12 , a significant improvement in IGA appeared in the fezakinumab group compared with placebo-treated patients, and progressive improvements lasted until week 20. Common adverse events included upper respiratory tract infections. This trial had a limited sample size and a lack of assessment in EASI and pruritus evaluation. More studies should be conducted in the future to evaluate the safety, dose strategy, and long-term efficacy in AD treatment.

\section{Anti-IL-33 Antibodies and Etokimab}

IL-33 is an alarmin cytokine produced by fibroblasts, epithelial, endothelial, and hematopoietic cells. It can be rapidly released from damaged cells in response to stress conditions such as infection, injury, and inflammation, and is recognized as a critical candidate to control atopic disorders [75]. A study has shown that the IL-33/ST2 pathway contributes to inflammation associated with disorders, including $\mathrm{AD}$. It initiated an adaptive type $2 \mathrm{im}$ mune response characterized by the production of IL-4, IL-5, and IL-13 [76, 77].

A phase $2 \mathrm{a}$ trial with etokimab, a humanized $\operatorname{IgG}_{1} /$ kappa anti-IL-33 monoclonal antibody, was performed with 12 moderate-to-severe adult $\mathrm{AD}$ patients [78]. Etokimab was given as an SD of $300 \mathrm{mg}$ intravenously. Twelve patients were observed for 140 days. All 12 patients achieved at least EASI-50 after an SD of etokimab, and 3 patients achieved IGA 0/1.

In summary, etokimab is at the initial investigational stage, and trials in a limited sample size suggest it is an efficient biologic for AD. More studies with larger sample sizes are needed, and safety issues should be further investigated in the future.

\section{TSLP and Tezepelumab}

TSLP is an epithelial cell-derived cytokine produced in response to proinflammatory stimuli. TSLP-activated dendritic cells induced the production of Th2 cytokines, including IL-4, IL-5, and IL-13 [79]. Thus, TSLP could be a key target to control AD-associated skin inflammation. Tezepelumab (AMG157/MEDI9929) is a human immunoglobulin G2 $\lambda$ monoclonal antibody that binds TSLP.

Simpson et al. [80] performed a phase 2 a study in which 113 moderate-to-severe $\mathrm{AD}$ patients were randomized 1:1 to tezepelumab $280 \mathrm{mg}$ or placebo Q2W sub- 
cutaneously with TCS. The percentage of EASI-50 was evaluated in week 12 . As a result, patients treated with tezepelumab plus TCS presented a greater percentage of EASI-50 compared to the placebo plus TCS group. In week 16, further improvement had also been achieved. The trial presented a novel biologic method for AD treatment but with a limited sample. A further trial with tezepelumab as monotherapy should be conducted in the future.

\section{OX40 Antibody and GBR830}

OX40 (CD134) is a costimulatory molecule of the TNF family, predominately expressed on T cells [81]. OX40OX40L interaction bridges the Th2 and Th1 pathways and increases cytokine production [82]. A study has shown that the numbers of OX40L+ DCs are highly increased in $\mathrm{AD}$ patients with greater OX40 expression in $A D$ lesions [83]. GBR830 is an investigational humanized $\mathrm{IgG}_{1} \mathrm{mAb}$ specific for inhibiting OX40 to treat autoimmune and chronic inflammatory disorders.

A phase 2 a study was performed to investigate the safety and efficacy of GBR830 in AD patients [84]. Sixty-one moderate-to-severe $\mathrm{AD}$ patients were randomized $3: 1$ to $10 \mathrm{mg} / \mathrm{kg}$ GBR830 intravenously or placebo on day 1 and day 29. Biopsy specimens were collected on days 1, 29, and 71 to evaluate the change of disease activities along with biomarkers like epidermal hyperplasia or cytokines. On day 71, more patients showed greater improvement in EASI with GBR830 treatment. Significant reductions on mRNA of cytokines like IL-31, CCL11, CCL17, and S100 were demonstrated. Hyperplasia measures of thickness, keratin 16, and Ki67 showed more significant reductions with GBR830. The above study suggests another possible target for $\mathrm{AD}$ patients as well as a novel therapeutic method. More clinical trials should focus on larger samples and the safety of GBR830.

\section{$\mathrm{H}_{4} \mathrm{R}$-Antagonist and JNJ-39758979}

$\mathrm{H}_{4} \mathrm{R}$ (histamine $\mathrm{H}_{4}$ receptor) has been identified to play a role in inflammatory responses [85]. $\mathrm{H}_{4} \mathrm{R}+$ expression of CD4+ $\mathrm{T}$ cells tends to be higher in patients with $\mathrm{AD}$ versus healthy patients [86]. The proliferation of keratinocytes from $\mathrm{AD}$ patients is increased on the activation of the $\mathrm{H}_{4} \mathrm{R}$ [87]. JNJ-39758979 is a selective, orally active $\mathrm{H}_{4} \mathrm{R}$ antagonist that inhibited the itch sensation induced by intradermal injection of histamine in healthy human subjects [88].

A total of 87 patients received 100 or $300 \mathrm{mg}$ of JNJ39758979 or placebo once daily for 6 weeks in a phase $2 a$ clinical trial [89]. Improvements in EASI and IGA were recorded to evaluate the efficacy. The results were recorded in week 6 , and the changes in EASI scores from baseline were greater in the 100- and 300-mg groups versus placebo. Respectively, 6.7 and $5.9 \%$ of patients receiving the 100- and 300-mg doses achieved IGA 0/1. Although it did not meet the primary endpoint, numerical improvement in EASI should be noted as well as the effect on controlling pruritis.

In summary, the application of the $\mathrm{H}_{4} \mathrm{R}$ antagonist and JNJ-39758979 in AD is a noteworthy trial. The findings suggest it may be beneficial for $\mathrm{AD}$, particularly in controlling pruritus. The trial was performed only on Japanese people, and further studies need to be completed for the safety and dose strategy.

\section{Conclusion}

Many biological therapeutic methods are currently used in $\mathrm{AD}$ treatment. Some of them are being studied in phase 2-3 clinical trials. Studies have shown the efficacy of these therapies in the treatment of AD. However, some of the relative novel biologics are only at an initial investigational stage. Other biologics, such as oclacitinib, PF04965842, and ASN002, were not included in this review due to a lack of clinical trials or only being reported in cases. Further understanding of the pathogenesis of $\mathrm{AD}$ could orient the investigational direction of future target therapies.

Moreover, biologics enrolled in this review were mainly given subcutaneously, intravenously, orally, and topically. Studies exploring better drug delivery methods should also be conducted in future studies. Considering the higher bioavailability and better dose-control ability, biologics with carriers like nanocarriers and nanogels may show better efficacy. Given that most nanotechnology studies are still at an in vitro or in vivo stage, they offer a promising study direction for $\mathrm{AD}$ treatment.

As many patients are increasingly interested in biologics, and more dermatologists choose biologics as their first-line therapeutic plan, the present review will help clinicians make the recommendation to patients who are either uninterested in the conventional approach or who would like to try a biologic therapy. Although larger, welldesigned, controlled studies or head-to-head studies are needed to continue testing the efficacy and safety of some of the biologics mentioned in this paper, they will possibly become the future study direction and main force in $\mathrm{AD}$ treatment. 


\section{Key Message}

This review summarizes and offers robust evidence for atopic dermatitis biological therapies.

\section{Conflict of Interest Statement}

The authors have no conflicts of interest to declare.

\section{Funding Sources}

This work was funded by the Military Medical Science and Technology Youth Cultivation Program (No. 19QNP113).

\section{Author Contributions}

S.Z. and F.Q. did the conception and design work. S.Z. and B.Z. searched the articles. S.Z. and F.Q. reviewed the literature. S.Z., B.Z., and F.Q. retrieved the articles. S.Z. and F.Q. did the acquisition and analysis. S.Z. and B.Z. interpretated the data. S.Z. and F.Q. wrote thes paper. Y.G. and F.Q. revising the draft. J.Z. and B.Z. offered the funds. F.Q. gave final approval of the version to be published.

\section{References}

1 Barbarot S, Auziere S, Gadkari A, Girolomoni G, Puig L, Simpson EL, et al. Epidemiology of atopic dermatitis in adults: results from an international survey. Allergy. 2018 Jun;73(6): 1284-93.

2 Flohr C, Mann J. New insights into the epidemiology of childhood atopic dermatitis. Allergy. 2014 Jan;69(1):3-16.

3 Drucker AM, Ellis A, Jabbar-Lopez Z, Yiu ZZ, Arents BW, Burton T, et al. Systemic immunomodulatory treatments for atopic dermatitis: protocol for a systematic review with network meta-analysis. BMJ Open. 2018 Aug; 8(8):e023061.

4 Laughter MR, Maymone MB, Karimkhani C, Rundle C, Hu S, Wolfe S, et al. The Burden of Skin and Subcutaneous Diseases in the United States From 1990 to 2017. JAMA Dermatol. 2020 Aug;156(8):874-81.

5 Langan SM, Irvine AD, Weidinger S. Atopic dermatitis. Lancet. 2020 Aug;396(10247): 345-60.

6 Drucker AM, Ellis AG, Bohdanowicz M, Mashayekhi S, Yiu ZZ, Rochwerg B, et al. Systemic Immunomodulatory Treatments for Patients With Atopic Dermatitis: A Systematic Review and Network Meta-analysis. JAMA Dermatol. 2020 Jun;156(6):659-67.

7 Fargnoli MC, Esposito M, Ferrucci S, Girolomoni G, Offidani A, Patrizi A, et al.; Dupilumab Italian National Access Program (DupINAP group). Real-life experience on effectiveness and safety of dupilumab in adult patients with moderate-to-severe atopic dermatitis. J Dermatolog Treat. 2019 Oct;1-7.

8 Barrett M, Luu M. Differential Diagnosis of Atopic Dermatitis. Immunol Allergy Clin North Am. 2017 Feb;37(1):11-34.

9 van der Schaft J, Thijs JL, Garritsen FM, Balak D, de Bruin-Weller MS. Towards personalized treatment in atopic dermatitis. Expert Opin Biol Ther. 2019 May;19(5):469-76.

10 Damiani G, Eggenhöffner R, Pigatto PD, Bragazzi NL. Nanotechnology meets atopic dermatitis: current solutions, challenges and future prospects. Insights and implications from a systematic review of the literature. Bioact Mater. 2019 Dec;4:380-6.
11 Moher D, Liberati A, Tetzlaff J, Altman DG; PRISMA Group. Preferred reporting items for systematic reviews and meta-analyses: the PRISMA statement. J Clin Epidemiol. 2009 Oct;62(10):1006-12.

12 Noda S, Krueger JG, Guttman-Yassky E. The translational revolution and use of biologics in patients with inflammatory skin diseases. J Allergy Clin Immunol. 2015 Feb;135(2):324-36.

13 Leung DY, Guttman-Yassky E. Deciphering the complexities of atopic dermatitis: shifting paradigms in treatment approaches. J Allergy Clin Immunol. 2014 Oct;134(4):769-79.

14 Hamilton JD, Suárez-Fariñas M, Dhingra N, Cardinale I, Li X, Kostic A, et al. Dupilumab improves the molecular signature in skin of patients with moderate-to-severe atopic dermatitis. J Allergy Clin Immunol. 2014 Dec; 134(6):1293-300.

15 Gooderham MJ, Hong HC, Eshtiaghi P, Papp KA. Dupilumab: A review of its use in the treatment of atopic dermatitis. J Am Acad Dermatol. 2018 Mar;78(3 Suppl 1):S28-36.

16 Beck LA, Thaçi D, Hamilton JD, Graham NM, Bieber T, Rocklin R, et al. Dupilumab treatment in adults with moderate-to-severe atopic dermatitis. N Engl J Med. 2014 Jul; 371(2):130-9.

17 Kakinuma T, Nakamura K, Wakugawa M, Mitsui H, Tada Y, Saeki H, et al. Thymus and activation-regulated chemokine in atopic dermatitis: serum thymus and activation-regulated chemokine level is closely related with disease activity. J Allergy Clin Immunol. 2001 Mar;107(3):535-41.

18 Halling AS, Loft ND, Silverberg JI, GuttmanYassky E, Thyssen JP. Real-world evidence of dupilumab efficacy and risk of adverse events: a systematic review and meta-analysis. J Am Acad Dermatol. 2021 Jan;84(1):139-47.

19 Ferrucci S, Casazza G, Angileri L, Tavecchio S, Germiniasi F, Berti E, et al. Clinical Response and Quality of Life in Patients with Severe Atopic Dermatitis Treated with Dupilumab: A Single-Center Real-Life Experience. J Clin Med. 2020 Mar;9(3):791.

20 Deleuran M, Thaçi D, Beck LA, de BruinWeller M, Blauvelt A, Forman S, et al. Dupil- umab shows long-term safety and efficacy in patients with moderate to severe atopic dermatitis enrolled in a phase 3 open-label extension study. J Am Acad Dermatol. 2020 Feb; 82(2):377-88.

21 Cork MJ, Thaçi D, Eichenfield LF, Arkwright PD, Hultsch T, Davis JD, et al. Dupilumab in adolescents with uncontrolled moderate-tosevere atopic dermatitis: results from a phase IIa open-label trial and subsequent phase III open-label extension. Br J Dermatol. 2020 Jan;182(1):85-96.

22 Cork MJ, Thaçi D, Eichenfield LF, Arkwright PD, Sun X, Chen Z, et al. Dupilumab provides favourable long-term safety and efficacy in children aged $\geq 6$ to 12 years with uncontrolled severe atopic dermatitis: results from an open-label phase IIa study and subsequent phase III open-label extension study. $\mathrm{Br}$ J Dermatol. 2020; DOI: 10.1111/bjd.18476.

23 Ultsch M, Bevers J, Nakamura G, Vandlen R, Kelley RF, Wu LC, et al. Structural basis of signaling blockade by anti-IL-13 antibody Lebrikizumab. J Mol Biol. 2013 Apr;425(8): 1330-9.

24 Song CH, Lee JK. Lebrikizumab treatment in adults with asthma. N Engl J Med. 2011 Dec; 365(25):2432

25 Simpson EL, Flohr C, Eichenfield LF, Bieber T, Sofen H, Taïeb A, et al. Efficacy and safety of lebrikizumab (an anti-IL-13 monoclonal antibody) in adults with moderate-to-severe atopic dermatitis inadequately controlled by topical corticosteroids: A randomized, placebo-controlled phase II trial (TREBLE). J Am Acad Dermatol. 2018 May;78(5):863-871.e11.

26 Guttman-Yassky E, Blauvelt A, Eichenfield LF, Paller AS, Armstrong AW, Drew J, et al. Efficacy and Safety of Lebrikizumab, a HighAffinity Interleukin 13 Inhibitor, in Adults With Moderate to Severe Atopic Dermatitis: A Phase 2b Randomized Clinical Trial. JAMA Dermatol. 2020 Apr;156(4):411-20.

27 Eli Lilly and Company, Dermira Inc. Longterm safety and efficacy study of lebrikizumab (LY3650150) in participants with moderateto-severe atopic dermatitis. ClinicalTrials. gov, NCT04392154. 
28 Hoffmann-La Roche. A study to evaluate the safety of lebrikizumab compared to topical corticosteroids in adult patients with atopic dermatitis. ClinicalTrials.gov, NCT02465606.

29 May RD, Monk PD, Cohen ES, Manuel D, Dempsey F, Davis NH, et al. Preclinical development of CAT-354, an IL-13 neutralizing antibody, for the treatment of severe uncontrolled asthma. Br J Pharmacol. 2012 May; 166(1):177-93.

30 Wollenberg A, Howell MD, Guttman-Yassky E, Silverberg JI, Kell C, Ranade K, et al. Treatment of atopic dermatitis with tralokinumab, an anti-IL-13 mAb. J Allergy Clin Immunol. 2019 Jan;143(1):135-41.

31 Pharma LEO: Tralokinumab monotherapy for moderate to severe atopic dermatitis - ECZTRA 2 (ECZema TRAlokinumab Trial No. 2). 2018.

32 O'Shea JJ, Plenge R. JAK and STAT signaling molecules in immunoregulation and immune-mediated disease. Immunity. 2012 Apr;36(4):542-50.

33 Cotter DG, Schairer D, Eichenfield L. Emerging therapies for atopic dermatitis: JAK inhibitors. J Am Acad Dermatol. 2018 Mar;78(3 Suppl 1):S53-62.

34 Carey AJ, Tan CK, Ulett GC. Infection-induced IL-10 and JAK-STAT: A review of the molecular circuitry controlling immune hyperactivity in response to pathogenic microbes. JAK-STAT. 2012 Jul;1(3):159-67.

35 Ciechanowicz P, Rakowska A, Sikora M, Rudnicka L. JAK-inhibitors in dermatology: current evidence and future applications. J Dermatolog Treat. 2019 Nov;30(7):648-58.

36 Meyer DM, Jesson MI, Li X, Elrick MM, Funckes-Shippy CL, Warner JD, et al. Antiinflammatory activity and neutrophil reductions mediated by the JAK1/JAK3 inhibitor, CP-690,550, in rat adjuvant-induced arthritis. J Inflamm. 2010 Aug;7(1):41.

37 Krueger J, Clark JD, Suárez-Fariñas M, Fuentes-Duculan J, Cueto I, Wang CQ, et al.; A3921147 Study Investigators. Tofacitinib attenuates pathologic immune pathways in patients with psoriasis: A randomized phase 2 study. J Allergy Clin Immunol. 2016 Apr; 137(4):1079-90.

38 Papp KA, Menter A, Strober B, Langley RG, Buonanno M, Wolk R, et al. Efficacy and safety of tofacitinib, an oral Janus kinase inhibitor, in the treatment of psoriasis: a Phase $2 \mathrm{~b}$ randomized placebo-controlled dose-ranging study. Br J Dermatol. 2012 Sep;167(3):668-77.

39 Bissonnette R, Iversen L, Sofen $\mathrm{H}$, Griffiths CE, Foley P, Romiti R, et al. Tofacitinib withdrawal and retreatment in moderate-to-severe chronic plaque psoriasis: a randomized controlled trial. Br J Dermatol. 2015;172(5):1395-406.

40 Bachelez H, van de Kerkhof PC, Strohal R, Kubanov A, Valenzuela F, Lee JH, et al.; OPT Compare Investigators. Tofacitinib versus etanercept or placebo in moderate-to-severe chronic plaque psoriasis: a phase 3 randomised non-inferiority trial. Lancet. 2015 Aug;386(9993):552-61.
41 Papp KA, Menter MA, Abe M, Elewski B, Feldman SR, Gottlieb AB, et al.; OPT Pivotal 1 and OPT Pivotal 2 investigators. Tofacitinib, an oral Janus kinase inhibitor, for the treatment of chronic plaque psoriasis: results from two randomized, placebo-controlled, phase III trials. Br J Dermatol. 2015 Oct; 173(4):949-61.

42 Bissonnette R, Papp KA, Poulin Y, Gooderham M, Raman M, Mallbris L, et al. Topical tofacitinib for atopic dermatitis: a phase IIa randomized trial. Br J Dermatol. 2016 Nov; 175(5):902-11.

43 Shi JG, Chen X, Lee F, Emm T, Scherle PA, Lo $\mathrm{Y}$, et al. The pharmacokinetics, pharmacodynamics, and safety of baricitinib, an oral JAK $1 / 2$ inhibitor, in healthy volunteers. J Clin Pharmacol. 2014 Dec;54(12):1354-61.

44 Guttman-Yassky E, Silverberg JI, Nemoto O, Forman SB, Wilke A, Prescilla R, et al. Baricitinib in adult patients with moderate-to-severe atopic dermatitis: A phase 2 parallel, double-blinded, randomized placebo-controlled multiple-dose study. J Am Acad Dermatol. 2019 Apr;80(4):913-921.e9.

45 Eli Lilly Company. A study of baricitinib (LY3009104) in participants with moderateto-severe atopic dermatitis. ClinicalTrials. gov, NCT02576938.

46 Eli Lilly Company, Incyte Corp. Study of baricitinib (LY3009104) in patients with moderate to severe atopic dermatitis. ClinicalTrials. gov, NCT03334422.

47 Eli Lilly and Company, Incyte Corp. A study of baricitinib (LY3009104) in combination with topical corticosteroids in adults with moderate to severe atopic dermatitis. ClinicalTrials.gov, NCT03733301.

48 Eli Lilly and Company, Incyte Corp. A longterm study of baricitinib (LY3009104) with topical corticosteroids in adults with moderate to severe atopic dermatitis that are not controlled with cyclosporine or for those who cannot take oral cyclosporine because it is not medically advisable. ClinicalTrials.gov, NCT03428100.

49 Eli Lilly and Company, Incyte Corp. A study of baricitinib (LY3009104) in children and adolescents with atopic dermatitis. ClinicalTrials.gov, NCT03952559.

50 Babon JJ, Lucet IS, Murphy JM, Nicola NA, Varghese LN. The molecular regulation of Janus kinase (JAK) activation. Biochem J. 2014 Aug;462(1):1-13.

51 Oetjen LK, Mack MR, Feng J, Whelan TM, Niu H, Guo CJ, et al. Sensory Neurons Co-opt Classical Immune Signaling Pathways to Mediate Chronic Itch. Cell. 2017 Sep;171(1): 217-228.e13

52 Gooderham MJ, Forman SB, Bissonnette R, Beebe JS, Zhang W, Banfield C, et al. Efficacy and Safety of Oral Janus Kinase 1 Inhibitor Abrocitinib for Patients With Atopic Dermatitis: A Phase 2 Randomized Clinical Trial. JAMA Dermatol. 2019 Dec;155(12):1371-9.

53 Simpson EL, Sinclair R, Forman S, Wollenberg A, Aschoff R, Cork M, et al. Efficacy and safety of abrocitinib in adults and adolescents with moderate-to-severe atopic dermatitis (JADE MONO-1): a multicentre, doubleblind, randomised, placebo-controlled, phase 3 trial. Lancet. 2020 Jul;396(10246):255-66.

54 Silverberg JI, Simpson EL, Thyssen JP, Gooderham M, Chan G, Feeney C, et al. Efficacy and Safety of Abrocitinib in Patients With Moderate-to-Severe Atopic Dermatitis: A Randomized Clinical Trial. JAMA Dermatol. 2020 Aug;156(8):863-73.

55 Guttman-Yassky E, Thaçi D, Pangan AL, Hong HC, Papp KA, Reich K, et al. Upadacitinib in adults with moderate to severe atopic dermatitis: 16-week results from a randomized, placebo-controlled trial. J Allergy Clin Immunol. 2020 Mar; 145(3):877-84

56 Kim BS, Howell MD, Sun K, Papp K, Nasir A, Kuligowski ME; INCB 18424-206 Study Investigators. Treatment of atopic dermatitis with ruxolitinib cream (JAK1/JAK2 inhibitor) or triamcinolone cream. J Allergy Clin Immunol. 2020 Feb;145(2):572-82.

57 Incyte Corp. TRuE AD1 - an efficacy and safety study of ruxolitinib cream in adolescents and adults with atopic dermatitis. ClinicalTrials.gov, NCT03745638.

58 Incyte Corp. TRuE AD2 - an efficacy and safety study of ruxolitinib cream in adolescents and adults with atopic dermatitis. ClinicalTrials.gov, NCT03745651.

59 Incyte Corp. A pharmacokinetic study of ruxolitinib phosphate cream in pediatric subjects with atopic dermatitis. ClinicalTrials.gov, NCT03257644.

60 Tanimoto A, Ogawa Y, Oki C, Kimoto Y, Nozawa K, Amano W, et al. Pharmacological properties of JTE-052: a novel potent JAK inhibitor that suppresses various inflammatory responses in vitro and in vivo. Inflamm Res. 2015 Jan;64(1):41-51.

61 Tanimoto A, Shinozaki Y, Yamamoto Y, Katsuda Y, Taniai-Riya E, Toyoda K, et al. A novel JAK inhibitor JTE-052 reduces skin inflammation and ameliorates chronic dermatitis in rodent models: comparison with conventional therapeutic agents. Exp Dermatol. 2018 Jan; 27(1):22-9.

62 Amano W, Nakajima S, Kunugi H, Numata Y, Kitoh A, Egawa G, et al. The Janus kinase inhibitor JTE-052 improves skin barrier function through suppressing signal transducer and activator of transcription 3 signaling. J Allergy Clin Immunol. 2015 Sep;136(3):667-677.e7.

63 Yamamoto Y, Otsuka A, Nakashima C, Amano W, Tanimoto A, Hayashi M, et al. The effect of Janus kinase inhibitor on pruritus in an atopic dermatitis murine model. J Invest Dermatol. 2016;136(5):S92.

64 Nakagawa H, Nemoto O, Igarashi A, Saeki H, Kaino H, Nagata T. Delgocitinib ointment, a topical Janus kinase inhibitor, in adult patients with moderate to severe atopic dermatitis: A phase 3, randomized, double-blind, vehicle-controlled study and an open-label, long-term extension study. J Am Acad Dermatol. 2020 Apr;82(4):823-31. 
65 Nakagawa H, Nemoto O, Igarashi A, Saeki H, Murata R, Kaino H, et al. Long-term safety and efficacy of delgocitinib ointment, a topical Janus kinase inhibitor, in adult patients with atopic dermatitis. J Dermatol. 2020 Feb; 47(2):114-20

66 Nakagawa H, Nemoto O, Igarashi A, Saeki H, Oda M, Kabashima K, et al. Phase 2 clinical study of delgocitinib ointment in pediatric patients with atopic dermatitis. J Allergy Clin Immunol. 2019 Dec;144(6):1575-83.

67 Bilsborough J, Leung DY, Maurer M, Howell $\mathrm{M}$, Boguniewicz M, Yao L, et al. IL-31 is associated with cutaneous lymphocyte antigenpositive skin homing $\mathrm{T}$ cells in patients with atopic dermatitis. J Allergy Clin Immunol. 2006 Feb;117(2):418-25.

68 Szegedi K, Kremer AE, Kezic S, Teunissen $\mathrm{MB}$, Bos JD, Luiten RM, et al. Increased frequencies of IL-31-producing T cells are found in chronic atopic dermatitis skin. Exp Dermatol. 2012 Jun;21(6):431-6.

69 Cornelissen C, Lüscher-Firzlaff J, Baron JM, Lüscher B. Signaling by IL-31 and functional consequences. Eur J Cell Biol. 2012 Jun-Jul; 91(6-7):552-66.

70 Ruzicka T, Hanifin JM, Furue M, Pulka G, Mlynarczyk I, Wollenberg A, et al. Anti-interleukin-31 receptor A antibody for atopic dermatitis. N Engl J Med. 2017 Mar;376(9):82635.

71 Mihara R, Kabashima K, Furue M, Nakano M, Ruzicka T. Nemolizumab in moderate to severe atopic dermatitis: an exploratory analysis of work productivity and activity impairment in a randomized phase II study. J Dermatol. 2019 Aug;46(8):662-71.

72 Silverberg JI, Pinter A, Pulka G, Poulin Y, Bouaziz JD, Wollenberg A, et al. Phase 2B randomized study of nemolizumab in adults with moderate-to-severe atopic dermatitis and severe pruritus. J Allergy Clin Immunol. 2020 Jan;145(1):173-82.

73 Lou H, Lu J, Choi EB, Oh MH, Jeong M, Barmettler S, et al. Expression of IL-22 in the Skin Causes Th2-Biased Immunity, Epidermal Barrier Dysfunction, and Pruritus via
Stimulating Epithelial Th2 Cytokines and the GRP Pathway. J Immunol. 2017 Apr;198(7): 2543-55.

74 Guttman-Yassky E, Brunner PM, Neumann AU, Khattri S, Pavel AB, Malik K, et al. Efficacy and safety of fezakinumab (an IL-22 monoclonal antibody) in adults with moderate-to-severe atopic dermatitis inadequately controlled by conventional treatments: A randomized, double-blind, phase $2 \mathrm{a}$ trial. J Am Acad Dermatol. 2018 May;78(5):872-881.e6.

75 Hardman C, Ogg G. Interleukin-33, friend and foe in type- 2 immune responses. Curr Opin Immunol. 2016 Oct;42:16-24.

76 Salimi M, Barlow JL, Saunders SP, Xue L, Gutowska-Owsiak D, Wang X, et al. A role for IL-25 and IL-33-driven type-2 innate lymphoid cells in atopic dermatitis. J Exp Med. 2013 Dec;210(13):2939-50.

77 Imai Y, Yasuda K, Sakaguchi Y, Haneda T, Mizutani H, Yoshimoto T, et al. Skin-specific expression of IL-33 activates group 2 innate lymphoid cells and elicits atopic dermatitislike inflammation in mice. Proc Natl Acad Sci USA. 2013 Aug;110(34):13921-6.

78 Chen YL, Gutowska-Owsiak D, Hardman CS, Westmoreland M, MacKenzie T, Cifuentes L, et al. Proof-of-concept clinical trial of etokimab shows a key role for IL-33 in atopic dermatitis pathogenesis. Sci Transl Med. 2019 Oct;11(515):11.

79 Gittler JK, Shemer A, Suárez-Fariñas M, Fuentes-Duculan J, Gulewicz KJ, Wang CQ, et al. Progressive activation of $\mathrm{T}(\mathrm{H}) 2 / \mathrm{T}(\mathrm{H}) 22$ cytokines and selective epidermal proteins characterizes acute and chronic atopic dermatitis. J Allergy Clin Immunol. 2012 Dec; 130(6):1344-54.

80 Simpson EL, Parnes JR, She D, Crouch S, Rees $\mathrm{W}$, Mo M, et al. Tezepelumab, an anti-thymic stromal lymphopoietin monoclonal antibody, in the treatment of moderate to severe atopic dermatitis: A randomized phase $2 \mathrm{a}$ clinical trial. J Am Acad Dermatol. 2019 Apr; 80(4):1013-21.

81 Chen M, Xiao X, Demirci G, Li XC. OX40 controls islet allograft tolerance in CD154 de- ficient mice by regulating FOXP3+ Tregs. Transplantation. 2008 Jun;85(11):1659-62.

82 Webb GJ, Hirschfield GM, Lane PJ. OX40, OX40L and Autoimmunity: a Comprehensive Review. Clin Rev Allergy Immunol. 2016 Jun;50(3):312-32.

83 Fujita $\mathrm{H}$, Shemer A, Suárez-Fariñas M, Johnson-Huang LM, Tintle S, Cardinale I, et al. Lesional dendritic cells in patients with chronic atopic dermatitis and psoriasis exhibit parallel ability to activate T-cell subsets. J Allergy Clin Immunol. 2011 Sep;128(3):574-82.e1-12.

84 Guttman-Yassky E, Pavel AB, Zhou L, Estrada YD, Zhang N, Xu H, et al. GBR 830, an anti-OX40, improves skin gene signatures and clinical scores in patients with atopic dermatitis. J Allergy Clin Immunol. 2019 Aug; 144(2):482-493.e7.

85 Walter M, Kottke T, Stark H. The histamine $\mathrm{H}_{4}$ receptor: targeting inflammatory disorders. Eur J Pharmacol. 2011 Oct;668(1-2):15.

86 Gutzmer R, Mommert S, Gschwandtner M, Zwingmann K, Stark H, Werfel T. The histamine $\mathrm{H} 4$ receptor is functionally expressed on $\mathrm{T}(\mathrm{H}) 2$ cells. J Allergy Clin Immunol. 2009 Mar;123(3):619-25.

87 Glatzer F, Gschwandtner M, Ehling S, Rossbach K, Janik K, Klos A, et al. Histamine induces proliferation in keratinocytes from patients with atopic dermatitis through the histamine 4 receptor. J Allergy Clin Immunol. 2013 Dec;132(6):1358-67.

88 Kollmeier A, Francke K, Chen B, Dunford PJ, Greenspan AJ, Xia Y, et al. The histamine $\mathrm{H}_{4}$ receptor antagonist, JNJ 39758979, is effective in reducing histamine-induced pruritus in a randomized clinical study in healthy subjects. J Pharmacol Exp Ther. 2014 Jul;350(1): 181-7.

89 Murata Y, Song M, Kikuchi H, Hisamichi K, Xu XL, Greenspan A, et al. Phase 2a, randomized, double-blind, placebo-controlled, multicenter, parallel-group study of a H4 R-antagonist (JNJ-39758979) in Japanese adults with moderate atopic dermatitis. J Dermatol. 2015 Feb;42(2):129-39. 